\title{
Biomolecular motor-driven microtubule translocation in the presence of shear flow: analysis of redirection behaviours
}

\author{
Taesung Kim ${ }^{1,3}$, Ming-Tse Kao ${ }^{2}$, Edgar Meyhöfer ${ }^{1,2}$ and \\ Ernest F Hasselbrink ${ }^{1,3}$ \\ ${ }^{1}$ Department of Mechanical Engineering, University of Michigan, 2350 Hayward Street, \\ Ann Arbor, MI 48109, USA \\ 2 Department of Biomedical Engineering, University of Michigan, 2200 Bonisteel Boulevard, \\ Ann Arbor, MI 48109, USA \\ E-mail: taesungk@umich.edu and efhass@umich.edu
}

Received 5 October 2006, in final form 20 November 2006

Published 15 December 2006

Online at stacks.iop.org/Nano/18/025101

\begin{abstract}
We suggest a concept for powering microfluidic devices with biomolecular motors and microtubules to meet the demands for highly efficient microfluidic devices. However, to successfully implement such devices, we require methods for active control over the direction of microtubule translocation. While most previous work has employed largely microfabricated passive mechanical patterns designed to guide the direction of microtubules, in this paper we demonstrate that hydrodynamic shear flow can be used to align microtubules translocating on a kinesin-coated surface in a direction parallel to the fluid flow. Our evidence supports the hypothesis that the mechanism of microtubule redirection is simply that drag force induced by viscous shear bends the leading end of a microtubule, which may be cantilevered beyond its kinesin supports. This cantilevered end deflects towards the flow direction, until it is subsequently bound to additional kinesins; as translocation continues, the process repeats until the microtubule is largely aligned with the flow, to a limit determined by random fluctuations created by thermal energy. We present statistics on the rate of microtubule alignment versus various strengths of shear flow as well as concentrations of kinesin, and also investigate the effects of shear flow on the motility.
\end{abstract}

(Some figures in this article are in colour only in the electronic version)

S] Supplementary data files are available from stacks.iop.org/Nano/18/025101

\section{Introduction}

Kinesins play an important role in vesicle transport and mitosis in cells. They move along microtubules and generate forces. Microtubules are one of the cytoplasmic filaments, consisting of $\alpha$ - and $\beta$-tubulin dimers [1]. These biomolecular motors have been known to generate about $6 \mathrm{pN}$ of force [2-5] and move along microtubules at a speed of about $1 \mu \mathrm{m} \mathrm{s}^{-1}[6,7]$ with about $50 \%$ efficiency in converting the chemical energy

\footnotetext{
3 Authors to whom any correspondence should be addressed.
}

from hydrolysis of ATP into mechanical work [1, 8]. These biomolecular motors and microtubule systems, in vitro, were introduced as novel prime movers for micro-total analysis systems ( $\mu$ TAS), because they showed exclusive potentials for nanoscopic, self-contained, and highly efficient $\mu$ TAS systems [9-12]. However, in gliding assays, microtubules translocate in random directions, which presents a challenge for creating technologies based on microtubule translocation.

Methods for controlling the direction of translocation of microtubules on kinesin-coated surfaces can be classified 


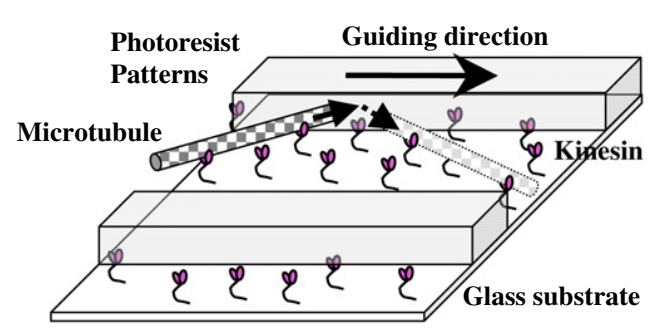

(a) Passive method

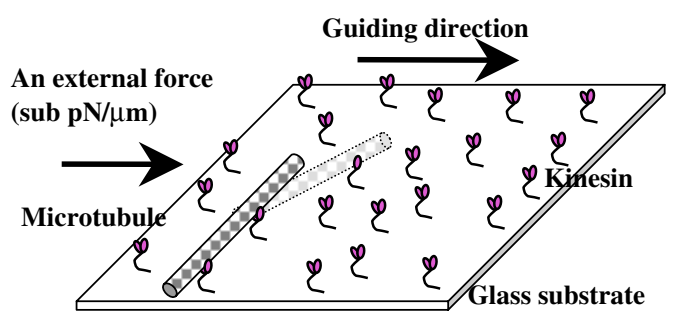

(b) Active method

Figure 1. Illustrations of mechanical passive methods and active methods. (a) Kinesin molecules are selectively adsorbed on a glass substrate at high density, but rarely on the photoresist patterns (at low density). The kinesins translocate the microtubule. The predetermined patterns confine the leading end of the microtubule, resulting in guiding the direction of the microtubule. (b) An external uniform load is applied to bend the leading end of the microtubule towards the parallel direction of the load. The remaining body of the microtubule is tightly clamped by kinesin bindings during the translocation from kinesin to kinesin, but the leading end of the microtubule bends like a cantilever beam subject to a load, resulting in alignment to the direction of the applied force.

as either passive or active. Passive methods generally use microfabrication or nanoimprinting technology to produce structural patterns. For example, as seen in figure 1(a), kinesins can be selectively immobilized on a glass surface using biocompatible surface patterns that confine translocation along certain tracks. When such patterned device structures are used for assays, the advancing ends of the microtubules run into walls of the patterns, bend towards the direction that requires less deflection, and then follow along the patterns or reflect out from the walls. Obviously, for this approach to be effective, it is fortunate that a single kinesin generates enough force to cause bending and even buckling of a microtubule [13]. To date, guiding microtubules along a straight line using chemical patterning or mechanical nanotracks [12, 14-20] and sorting them into one direction using arrow heads in microchannels have been demonstrated [18-20].

On the other hand, active methods exploit mechanical/electrical external forces such as viscous drag forces [11, 21-23] and electrophoretic forces [10, 19, 24, 25]. As seen in figure 1(b), the mechanism of these methods is assumed to be that the external forces bend the leading ends of microtubules in a desired direction to a certain degree, right before the advancing end is engaged to another proximate kinesin. However, the remainder appears to be fixed by kinesin bindings-at least as long as the applied forces are much less than those kinesins can generate (typically, a sub-pN force is applied on the microtubules by external forces, but a single ki- nesin can generate about $6 \mathrm{pN}$ and more than one kinesin can translocate a single microtubule simultaneously $[2,4,17])$.

Despite the potential of active methods, they have not been studied extensively in the past. We recently conducted a detailed study on the use of electric fields to achieve active control, and found the approach to be quite effective [24]. For example, clockwise translocation of a microtubule along a $30 \mu \mathrm{m}$ diameter circular trajectory was easily achieved using four electrodes that were used to rotate the applied electric field in a counterclockwise direction. However, integration of electrodes and electric control equipment into microfluidic devices might often not be portable. Since such microfluidic devices will require same procedure for introducing flows, using shear flows to steer microtubules might provide an attractive alternative. This motivated our investigation into the effects of simple hydrodynamic shear. In fact, microtubules have been observed to be aligned parallel to the flow direction during in vitro gliding assays as a result of repeatedly loading and flushing buffer solutions with kinesin and microtubule molecules into the microchannels. Furthermore, shear flow has been reported to make microtubules move parallel to the flow direction [23], and then has also been used to produce isopolar microtubule arrays to investigate the directionality of kinesin [22]. Recently, shear flow has been used to align microtubules parallel to the flow direction, followed by immobilization to provide pathways for kinesins to carry beads along with them in microchannels [11, 21].

The present work follows up on these qualitative findings by providing quantitative analysis of the governing parameters determining the rate of microtubule redirection/alignment in the presence of shear flow. Specifically, we explore the rate of microtubule alignment as functions of applied shear stress and the concentration of kinesin. The goal of this work is to provide quantitative understanding of microtubule translocation behaviour in vitro as well as in vivo, when shear stresses are present.

\section{Materials and methods}

\subsection{Kinesin and microtubule preparation}

For most experiments we used a bacterially expressed kinesin motor, NKHK560cys. This motor consists of the head and neck domain of Neurospara crassa kinesin (amino acids 1433) and stalk of Homo sapiens kinesin (residues 430-560) and a reactive cysteine at the C-terminal end [26]. Kinesin was expressed and purified as described previously [20, 27]. Tubulin was purified from cow brain by three cycles of microtubule polymerization and depolymerization followed by phosphocellulose ion exchange chromatography, and fluorescently labelled tubulin (TMR-tubilin) was prepared by reacting polymerized microtubules with a 20 -fold excess of tetramethylrhodamine (Molecular Probes) at room temperature for $30 \mathrm{~min}$. Labelled tubulin was purified from this mixture by repeated depolymerization and polymerization. For all experiments, microtubules were polymerized by incubating $2 \mathrm{mg} \mathrm{ml}^{-1}$ tubulin (equal ratios of TMR-labelled and unlabelled tubulin), $1 \mathrm{mM}$ GTP and $4 \mathrm{mM} \mathrm{MgCl}_{2}$ in BRB80 buffer at $37^{\circ} \mathrm{C}$ for $20 \mathrm{~min}$. Microtubules were stabilized by the addition of $10 \mu \mathrm{M}$ taxol. All motility assays were carried out in BRB80 buffer at $\mathrm{pH} 6.8$ at room temperature. 


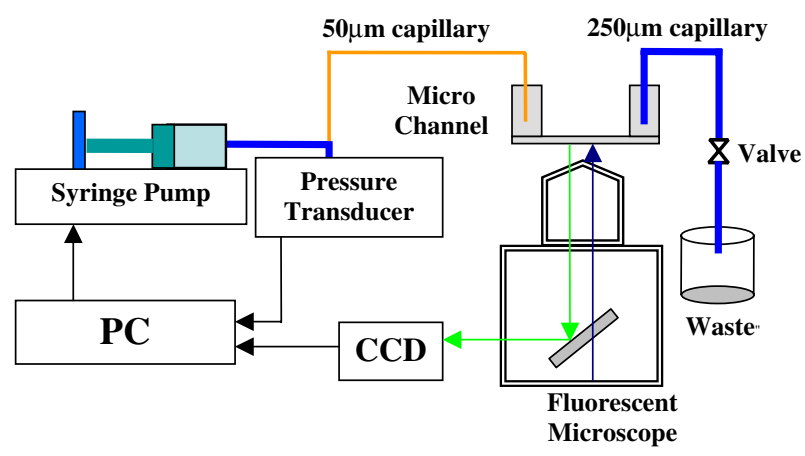

Figure 2. Schematic diagrams of the experimental setup.

\subsection{Experimental setup}

Figure 2 shows the experimental setup. This setup monitors the motility of microtubules by kinesin and controls shear stresses (flow rates) in microfluidic channels. Rhodamine-labelled microtubule motility is captured via a CCD camera (Orca ER, Hamamatsu, Japan) equipped with a fluorescent microscope (Axiovert 200, Carl Zeiss, Germany). Controlled flow can be generated in the microfluidic channels by using a highresistance capillary connected in series with a syringe pump (KDS210, KD Scientific, Holliston, MA, attainable pressure ranges from 0 to $250 \mathrm{psi}$ ), and by using feedback control from a pressure transducer (40PC250G2A, Honeywell).

\subsection{Microchannel fabrication}

Microfluidic channels were etched in $100 \mathrm{~mm}$ diameter glass substrates using standard HF wet-etching and lithography techniques. The channels are typically $100 \mu \mathrm{m}$ deep, $400 \mu \mathrm{m}$ wide, and $15 \mathrm{~mm}$ long. These bottom microfluidic channels were bonded to coverslips using a low-temperature SU-8 ( $T_{\mathrm{g}}=$ $75^{\circ} \mathrm{C}$ ) bonding technique shown in figure 3 , wherein a PDMS

1. HF-wet etching using photolithography

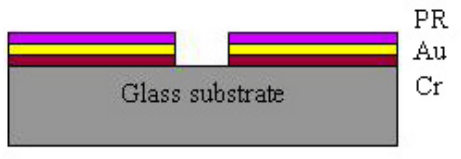

2. Electrochemical drilling for pores

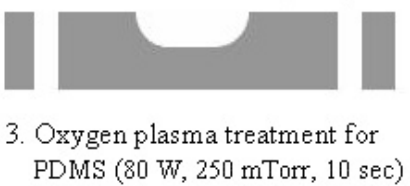
PDMS (80 W, $250 \mathrm{mTorr}, 10 \mathrm{sec})$

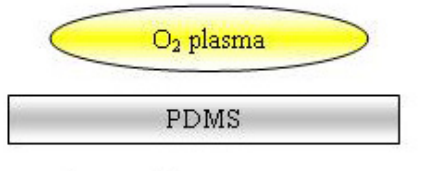

4. Spin coat of $500 \mathrm{~nm}$ SU-8 on PDMS

PDMS (polydimethylsiloxane) stamp is used for transferring SU-8 to the glass surface. The PDMS stamp is first treated with oxygen RIE (Reactive Ion Etching) for $10 \mathrm{~s}$ at $80 \mathrm{~W}$ and 250 mTorr to make the surface hydrophilic [28]. SU-8 2 (Microchem) is immediately poured on the PDMS surfaces and spun at $4000 \mathrm{rpm}$ for $30 \mathrm{~s}$ to obtain a coating of about $500 \mathrm{~nm}$ thickness. The glass microfluidic channels are stamped onto the top of the SU-8 layer and separated $30 \mathrm{~s}$ later, transferring the SU-8; the process is quite reliable because the $\mathrm{SU}-8$ has a much higher affinity for glass than for PDMS, and furthermore, the SU-8 only transfers where it is in direct contact with the glass (e.g., it does not fill the etched channels). The glass/SU8 is then cured on an $85^{\circ} \mathrm{C}$ hot plate for $2 \mathrm{~min}$ to remove the solvent. Bonding to a top coverslip $\left(22 \times 30 \times 0.17 \mathrm{~mm}^{3}\right.$, Corning) is done by applying pressure of $50 \mathrm{kPa}$ at $75^{\circ} \mathrm{C}$ for $2 \mathrm{~min}$ using a cleanroom press. This bonding procedure minimizes SU-8 exposure to the fluid, since most of the wetted surfaces are glass.

The glass substrates are pre-drilled before bonding using an electrochemical drilling method, wherein the glass is submerged in a $45 \% \mathrm{NaOH}$ bath and is drilled using a sharp electrode biased at $35 \mathrm{~V}$ (DC) relative to a sacrificial electrode in the bath. Nanoports (N-125S, Upchurch Scientific, Oak Harbor, WA) are connected on top of the drilled holes for external reservoirs for loading solutions.

\subsection{Shear flow control (wall shear stress $\tau_{\mathrm{w}}$ )}

Relatively low shear stresses are required in the microchannel for these experiments. This, in turn, requires very small flow rates and, as it turns out, very low pressure differences across the microchannel. In our microchannel (several tens of $\mu \mathrm{m}$ in depth, a few $\mathrm{cm}$ long), the required shear stresses can be generated by pressure differentials of the order of tens of $\mathrm{Pa}$. Such low pressures are hard to control using standard pressure/flow hardware from commercial vendors. Instead, one can connect a long microcapillary (e.g. $50 \mu \mathrm{m}$ in diameter and

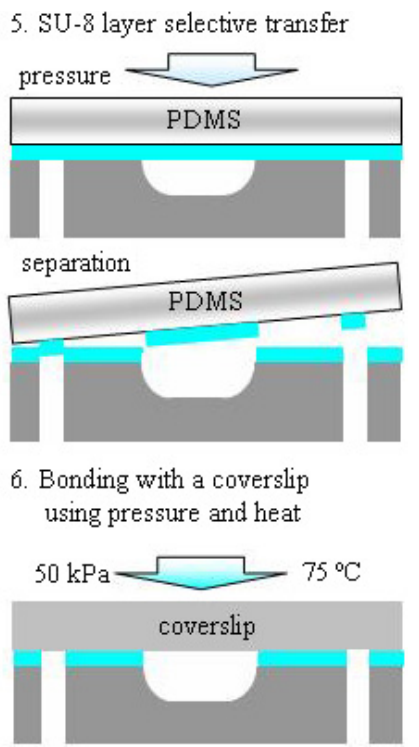

Figure 3. Procedures of microchannel fabrication. 
$1 \mathrm{~m}$ long, Polymicron Technologies, Phoenix, AZ) in series with the microchannel, so that the required pressure differential can be raised to a more easily managed range (even to hundreds of $\mathrm{kPa}$; see the Supporting Online Information available at stacks.iop.org/Nano/18/025101). As seen in figure 2, a syringe pump is operated in a feedback loop through a serial port of a PC, and the pressure values are acquired by the DAQboard and LabVIEW (PCI-6014, NI, Austin, TX). Flow rates are calibrated by measuring the weight of a fluid passing through the capillary and coming out from microchannels versus time with a digital balance (AL104, Mettler Toledo, North Brook, IL); flow rates are averaged over 10-30 min to reduce uncertainty. Further details and a typical calibration plot are shown in the Online Supporting Information available at stacks.iop.org/Nano/18/025101.

In this work, the wall shear stress was chosen to be a principal parameter to ascertain the external viscous loads on microtubules. This is because viscous loads are linearly proportional to the wall shear stress once the microtubules are translocating near a glass surface (in gliding assays, microtubules are approximately $21.5 \mathrm{~nm}$ above the surface [2]). However, the wall shear stress $\left(\tau_{\mathrm{w}}\right)$ in HF-etched microchannels varies along the periphery because the velocity profile is asymmetric. In other words, $\tau_{\mathrm{w}}$ at the bottom or top surface is higher than $\tau_{\mathrm{w}}$ at the side surfaces. To determine the uncertainty from the variance of the wall shear stress, as seen in figure 4(b), FLUENT (Fluent Inc., Lebanon, NH) simulations were conducted. The simulations show that $\tau_{\mathrm{w}}$ at the bottom surface (focus of view; see figure 4) is mostly uniform, except below the under-etched surfaces of the top surface, and slightly larger than the average wall shear stress, but no more than $10 \%$ larger. Therefore, in these experiments, the variance of $\tau_{\mathrm{w}}$ at the bottom surface is compensated for. The average wall shear stress is expressed with the cross-sectional area, entire surface area, and a pressure gradient as follows [29].

$$
\bar{\tau}_{\mathrm{w}}=\Delta P_{\mathrm{ch}} \frac{A_{x}}{A_{\mathrm{s}}} .
$$

Here, $\Delta P_{\mathrm{ch}}$ is the pressure gradient between the microchannel ends, $A_{x}$ is the cross-sectional area, and $A_{\mathrm{s}}$ is the entire surface area of the microchannel except the inlet and outlet areas. From the resistance analogy of the microchannel and the capillary (see Supporting Online Information available at stacks.iop.org/Nano/18/025101), the pressure between the microchannel and the capillary is divided as follows.

$$
\Delta P_{\mathrm{ch}}=\frac{R_{\mathrm{ch}}}{R_{\mathrm{cap}}} \Delta P_{\mathrm{syr}}
$$

By substituting equation (2) into equation (1), the average wall shear stress becomes

$$
\bar{\tau}_{\mathrm{w}}=\frac{R_{\mathrm{ch}}}{R_{\mathrm{cap}}} \frac{A_{x}}{A_{\mathrm{s}}} \Delta P_{\mathrm{syr}} .
$$

Therefore, this experimental setup makes it easy to generate nanolitre flow rates ranging from 0 to $250 \mathrm{nl} \mathrm{s}^{-1}$, which correspond to wall shear stress ranging from 0 to $2.0 \mathrm{~Pa}$. We estimate the uncertainty of the flow rate to be about $0.6 \mathrm{nl} \mathrm{s}^{-1}$ (limited by uncertainty in the pressure transducer reading), which corresponds to about $0.005 \mathrm{~Pa}$ uncertainty in the wall shear stress.
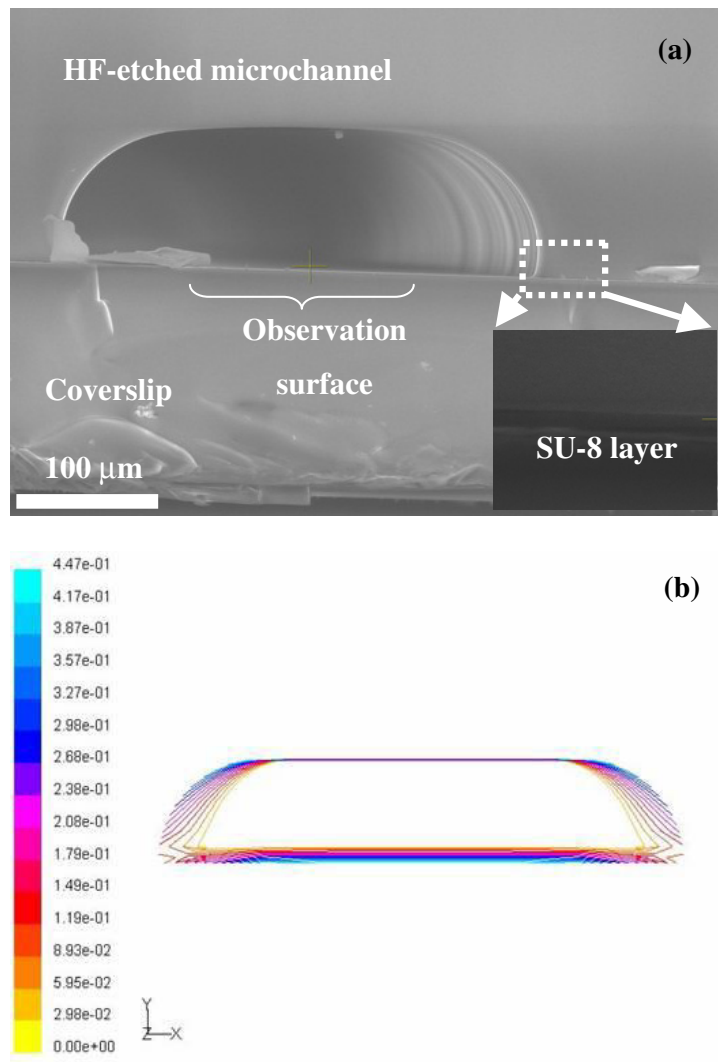

Figure 4. (a) A scanning electron microscope picture showing the cross section of the microchannel made of glass substrates and a microscope coverslip and then bonded with SU-8. Observation was made on the bottom where $\tau_{\mathrm{w}}$ is nearly constant. (b) FLUENT simulation result of wall shear stress distribution within the HF-etched microchannel. The bottom wall shows almost constant wall shear stress $\left(\tau_{\mathrm{w}}=0.45 \mathrm{~Pa}\right)$. The unit is the pascal.

\subsection{Surface concentration of kinesin}

In this work, we use two different kinesin surface densities. Unfortunately, a quantitative measurement of the functional kinesin surface densities cannot be easily provided; however, it is generally accepted that most adsorption isotherms are monotonic, and therefore the higher bulk concentration should result in a higher surface density. When we adsorb kinesin from a solution containing a concentration of $4.5 \mu \mathrm{g} \mathrm{ml}^{-1}$, assuming complete adsorption of kinesin to the microchannel, we expect a total surface density of about 2500 kinesins $\mu \mathrm{m}^{-2}$. Note that the number of adsorbed kinesins that are active and interact with the microtubule is much lower. Based on comparative experience with other kinesins, we know that these experimental conditions lead to surface densities that are just sufficient for continuous microtubule motility. Further dilution of kinesin results in an intermediate assay where microtubules are translocated for limited distance. Therefore, we refer to this concentration as a 'low'-density (concentration) assay. On the other hand, if we use $11.25 \mu \mathrm{g} \mathrm{ml}^{-1}$ of kinesin, the resulting surface density is about 2.5 times higher and we refer to this assay as a 'high'-density (concentration) assay. This range of kinesin concentrations showed significant variation in results. Each gliding assay was performed by using $100 \mu \mathrm{l}$ of kinesin 

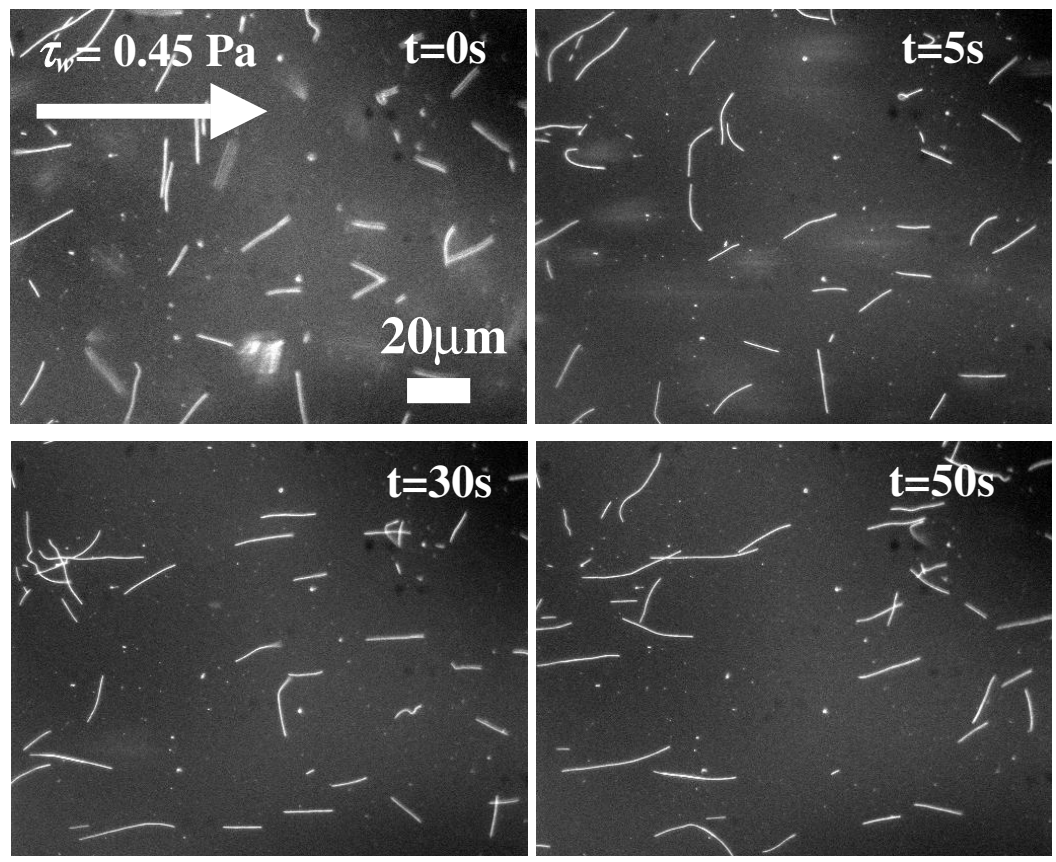

Figure 5. Gliding assay response to the application of shear flow $\left(\tau_{\mathrm{w}}=0.45 \mathrm{~Pa}\right)$. The series of pictures was captured at $5,30,50 \mathrm{~s}$ after shear flow was applied, respectively. Continuous application of a viscous drag force produces alignment of the microtubules parallel to the flow field at low concentration of kinesin. The arrow indicates the flow direction.

solution at different concentrations, allowing this to incubate for $5 \mathrm{~min}$. Subsequently, $100 \mu 1$ of $0.03 \mathrm{mg} \mathrm{ml}^{-1}$ microtubule solution was introduced. The standard buffer solutions used to generate shear flow contained $80 \mathrm{mM}$ of K.PIPES (to adjust the $\mathrm{pH}$ to 6.8$), 1 \mathrm{mM}$ of $\mathrm{MgCl}_{2}, 1 \mathrm{mM}$ of EGTA, $3 \mathrm{mM}$ of ATP, and a 'high' or 'low' concentration of kinesin as described above to reduce the unexpected detachment of microtubules.

It was also discovered in our previous study that surface preparation was very important in achieving highly repeatable results. Typically, gliding assays are conducted in glass chambers built with coverslips as received. However, it was found in our previous study that the surfaces of the glass coverslips may affect the adsorption of kinesin. Thus all microchannels were cleaned with piranha $\left(\mathrm{H}_{2} \mathrm{O}_{2}: \mathrm{H}_{2} \mathrm{SO}_{4}=1: 1\right)$ for 10 min, etched with 20 times diluted HF $(49 \%)$ for $10 \mathrm{~s}$, and rinsed with DI water for $10 \mathrm{~min}$. It was observed that the number of microtubules on treated glass surfaces at a low concentration of kinesin is comparable with that on untreated glass surfaces (as received) at a normal concentration [14]; from this it might be inferred that the surface treatment increases the surface density of kinesin.

\section{Results}

\subsection{Qualitative results}

Figure 5 shows the translocation of microtubules on kinesincoated glass surfaces in the presence of shear flow $\left(\tau_{\mathrm{w}}=\right.$ $0.45 \mathrm{~Pa}$ ). Initially, the microtubules are translocating in random directions, as observed in typical gliding assays. However, $5 \mathrm{~s}$ after the shear flow is applied, the advancing ends of the microtubules appear to bend towards the direction of the flow field. After $50 \mathrm{~s}$, nearly all the microtubules are translocating roughly parallel to the flow field.
Using these observations and the results of our previous work [24] as a guide, our hypothesis is that, although viscous drag is too weak to detach a microtubule from kinesin, the drag force is sufficient to deflect the leading end of the microtubule into the flow direction by an appreciable amount, as long as the leading end of the microtubule is cantilevered beyond its kinesin supports (e.g., for the period of time before the advancing tip engages another proximate kinesin). Since the

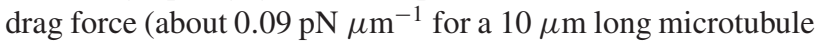
perpendicular to the flow direction under $\tau_{\mathrm{w}}=0.45 \mathrm{~Pa}$ ) is much less than the force a single kinesin can generate (about $6 \mathrm{pN}$ ), the only question is whether the drag force is sufficient to completely explain the observations qualitatively. This hypothesis has several testable corollaries. (1) the rate of microtubule alignment in the presence of shear flow should strongly depend on the surface concentration of active kinesin, because if we presume that microtubule deflection can be modelled much like a cantilever beam deflecting under a uniform loading, the amount of deflection is proportional to the length of the cantilever to the fourth power. (2) The rate of deflection should also increase with increasing wall shear stress, $\tau_{\mathrm{w}}$ (which is proportional to the flow rate/pressure gradient). (3) In low-kinesin-concentration cases, we should observe sharp bending at a few points along the length of a microtubule, corresponding to the location of kinesin supports, accompanied by sharp-turning trajectories. However, in highkinesin-concentration cases, the closely spaced kinesins will create a more gradual turning along gently arcing trajectories.

All three of these qualitative features are observed in our images, and, as we show in the following section, in our quantitative data. Qualitatively, figure 6 shows snapshots of microtubules at fixed times after the application of various shear stresses, and at two concentrations of bulk kinesin. 

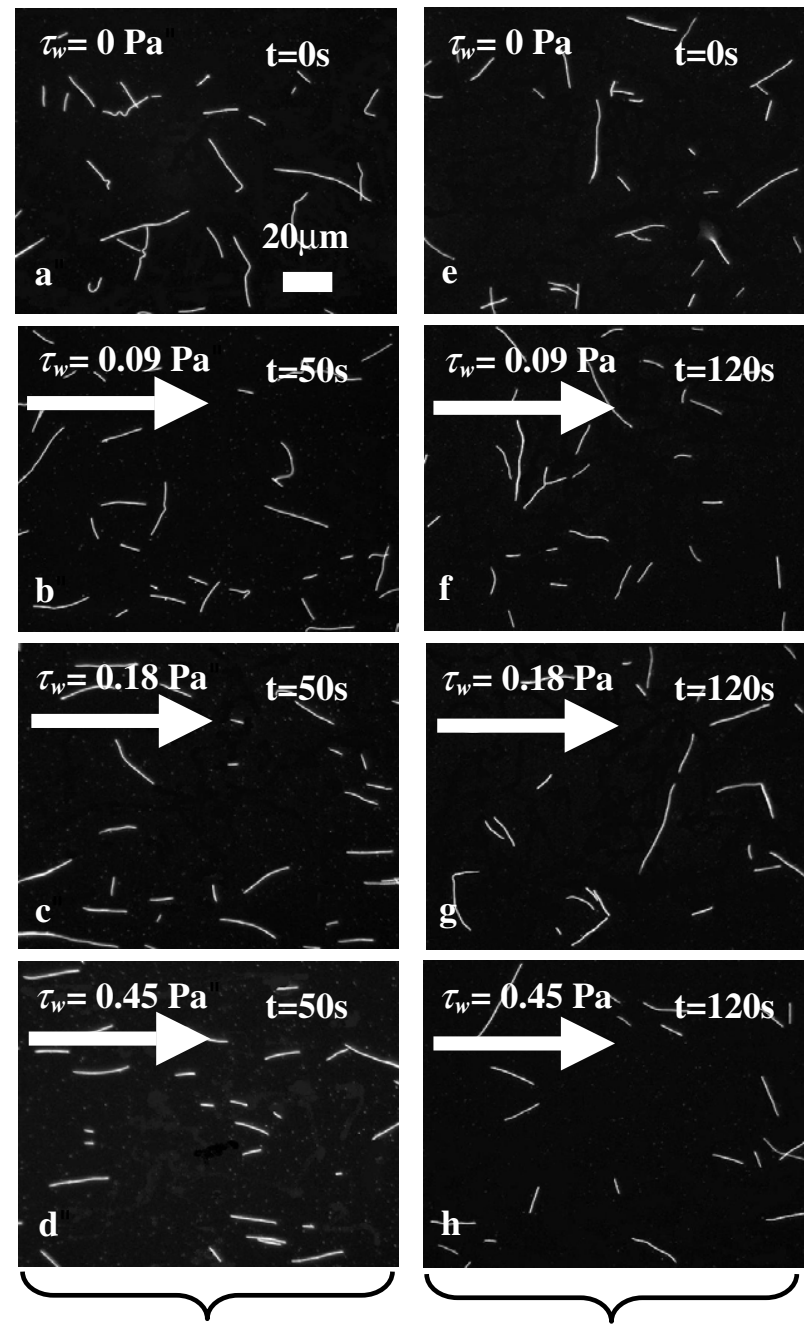

Low concentration
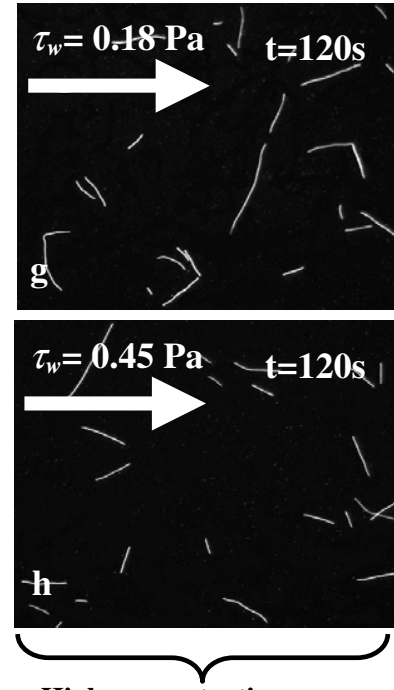

High concentration

Figure 6. Gliding assay response to the application of various wall shear stresses. (a) Low kinesin concentration, no wall shear stress (standard gliding assay). (b)-(d) Low-concentration kinesin gliding assays, after $50 \mathrm{~s}$ exposure to different wall shear stresses, such as $\tau_{\mathrm{w}}=0.09,0.18$, and $0.45 \mathrm{~Pa}$, respectively. (e) High kinesin concentration, no wall shear stress (standard gliding assay). (f)-(g) High-concentration kinesin gliding assays, after $120 \mathrm{~s}$ exposure to wall shear stresses, such as $\tau_{\mathrm{w}}=0.09,0.18$, and $0.45 \mathrm{~Pa}$, respectively.

The alignment of microtubules for the low-concentration case (figures 6(a)-(d)) is much more pronounced than in the highconcentration case (figures 6(e)-(h)), even though the highconcentration images are shown after a longer exposure to shear. It is noted that, in both cases, the buffer solution does not contain microtubules; therefore it is not surprising that the microtubule concentration on the surface slowly decreases due to the occasional microtubule detachment. Further analysis of images such as these will be presented in section 3.2. Figure 7 compares examples of individual microtubule trajectories on a low concentration of kinesin (low surface density of kinesin) and high concentration of kinesin (high surface density of kinesin). In figure 7(a), a microtubule is translocating upwards in the image, with its leading end bent slightly to the right, in the presence of a nearly perpendicular flow field $\left(\tau_{\mathrm{w}}=\right.$
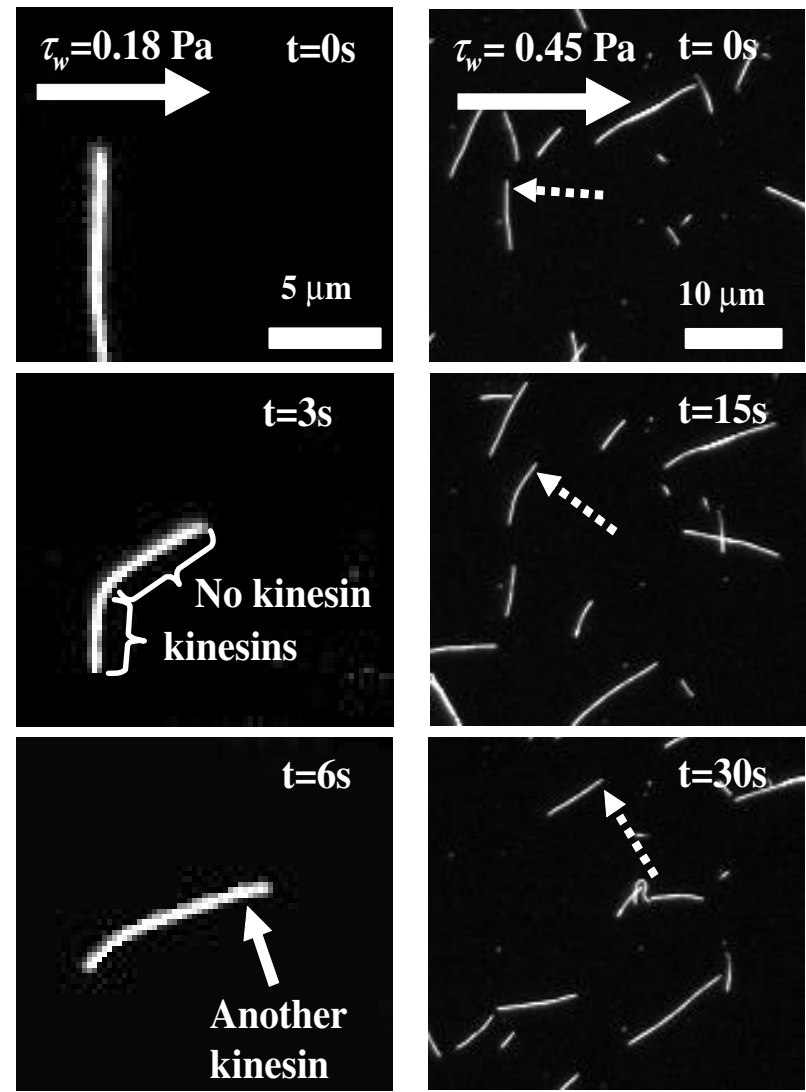

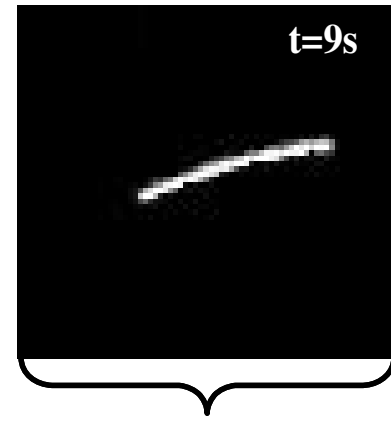

(a) Low concentration

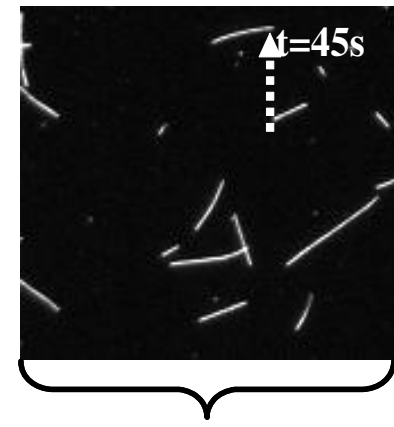

(b) High concentration
Figure 7. The process of aligning microtubules at different

concentrations of kinesin using shear flow. (a) A microtubule gliding on a low density of kinesin deflects like a cantilever beam subject to a load, showing significant deflection as one leading end advances until it is apparently bound to another kinesin. (b) A microtubule gliding on a high density of kinesin makes a slower and more gradual turn.

$0.18 \mathrm{~Pa}$ ). The leading end is observed to experience a large bending in a short distance. In contrast, high-kinesindensity cases show much more gradual alignment, as shown in figure 7(b), for instance. The microtubule indicated with a dotted arrow in figure 7(b) turns smoothly, with a large radius of curvature. Our interpretation of figure 7(a) (at $t=0$ and $3 \mathrm{~s}$ ) is that the microtubule seems to be transiently cantilevered over a region where the kinesin failed to engage the microtubule. We hypothesize that the cantilevered portion of the microtubule seems to be deflected towards the flow direction by the bending moment caused by a uniform transverse load, while the remainder was tightly fixed by active kinesins like a series 


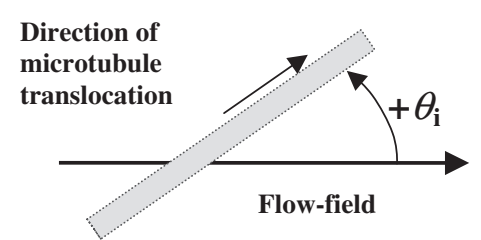

Figure 8. Definition of microtubule translocation direction $\theta_{i}\left(-180^{\circ}<\theta_{i}<180^{\circ}\right)$. Counterclockwise is positive.

of clamps along its length. The deflection gradually increases as the microtubule translocates, until the tip is engaged by another kinesin (figure 7(a), $t=6 \mathrm{~s}$ ), and the process repeats. As a side note, probabilistically, the longer and the more cantilevered microtubules are expected to be observed on the lower concentration of kinesin — and this is indeed the case.

\subsection{Quantitative results}

We use straightforward image processing techniques to analyse quantitatively the rate of microtubule alignment. Briefly, angles between the leading end of each microtubule in the video frame and the direction of the flow field, as shown in figure 8 , were measured in each video frame manually. The translocation direction $\theta_{i}$ is defined as the angle between the flow-field vector and a vector pointing in the direction of microtubule translocation.

Before considering aggregate statistical behaviour, let us consider the behaviour of some individual microtubules. In figure 9, representative histories of the angle $\theta_{i}$ (as defined in figure 8) for ten randomly chosen microtubules are plotted in the presence of three different wall shear stresses. The microtubules were chosen so that their initial $\theta_{i}$ were distributed evenly between $\pm \pi$. Figure 9(a) shows the values with low kinesin density, but figure 9(b) shows histories with high kinesin density. In all cases, obviously, shear flow appears to align all microtubules, but in the low-density cases, the alignment is more pronounced, and occurs much faster, than in the high-density cases, as supposed by the cantilever beam bending hypothesis.

We also obtained ensemble statistics for $N=50$ microtubules as a function of time. The average $\theta_{i}=0$ for both randomly oriented and perfectly aligned microtubules, so this is not a useful statistic; however, the root-mean-square (RMS) value $\theta_{\mathrm{rms}}$ has a value of $\theta_{\mathrm{rms}}=(\pi 2 / 3)^{1 / 2}=103.9^{\circ}$ for randomly oriented microtubules, while an ensemble of perfectly aligned microtubules will have $\theta_{\mathrm{rms}}=0.0^{\circ}$; thus we use $\theta_{\text {rms }}$ as a convenient measure of statistical alignment. In figure $10, \theta_{\mathrm{rms}}$ is plotted as functions of time under different shear stresses and different concentrations of kinesin. Figure 10(a) shows the results at low concentration of kinesin (corresponding to images of figures 6(a)-(d)). The $\theta_{\text {rms }}$ before shear flows are applied is quite close to $103.9^{\circ}$, as expected (error bars include sampling as well as estimated measurement errors). After shear flows are applied, $\theta_{\text {rms }}$ decreases with time and, consistent with figure 5, the rate of alignment is larger under stronger wall shear stress. Figure 10(b) shows the result at exactly the same conditions, except with high concentration of kinesin (corresponding to figures 6(e)-(h)). $\theta_{\text {rms }}$ decreases significantly faster at low concentration of kinesin than at high concentration when comparing results at similar wall shear stress. Again, these statistical results are also consistent with the hypothesis of a cantilever beam deflection described above.

\section{Discussion}

In this study, we conducted numerous gliding assays to investigate the translocation behaviours of microtubules on different surface densities of kinesin in the presence of various wall shear stresses. However, when shear flow was applied, the surface density of microtubules appeared to gradually decrease, and also microtubules were observed to be detached from the surfaces even under a very low shear rate-at which the average velocity is several tens of $\mu \mathrm{m} \mathrm{s}^{-1}$. Our calculations showed that this range of shear rate should result in forces far less than the $6 \mathrm{pN}$ that a kinesin can exert. A closer analysis suggested that this behaviour is related to reversal kinesin
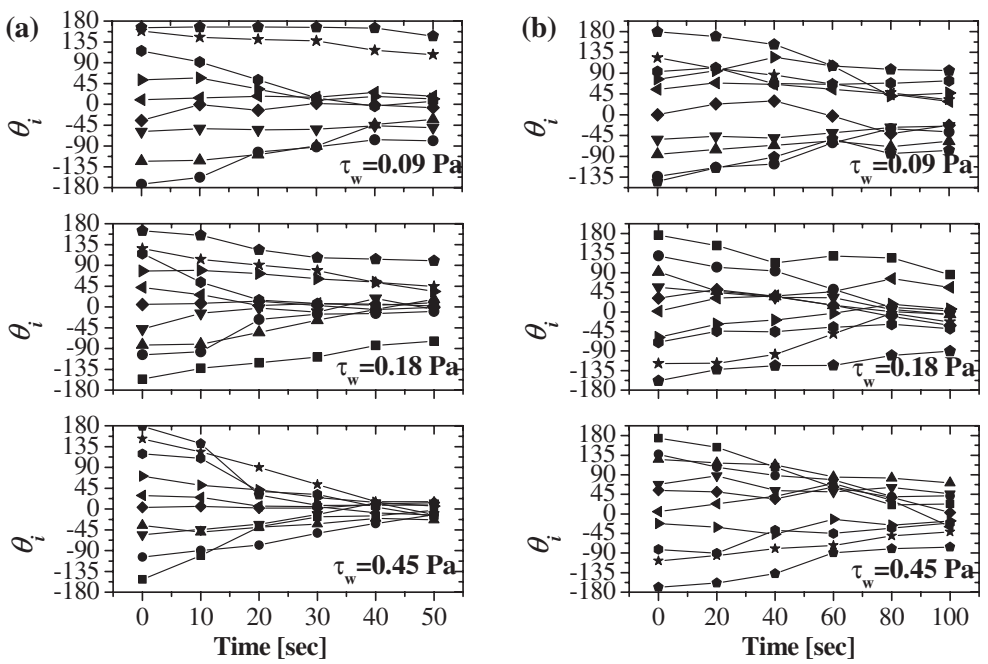

Figure 9. Individual microtubule directions $\theta_{i}$ versus time. (a) Low-density-kinesin cases show faster alignment at various shear stresses. $\theta_{i}$ for 10 cases are shown. (b) High-density-kinesin cases show slow and random alignment. 

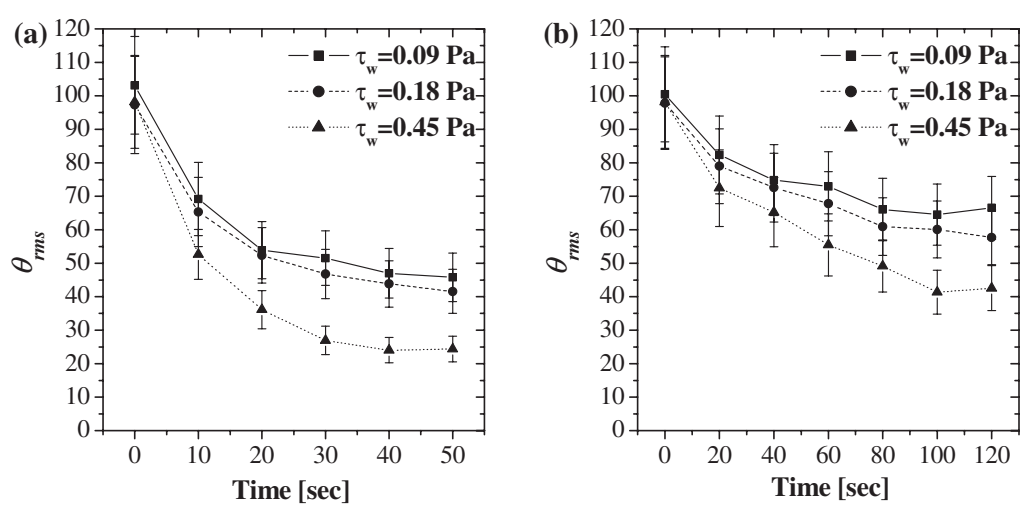

Figure 10. $\theta_{\mathrm{rms}}$ (directionality) of microtubules versus time $(N=50)$. (a) At low concentration of kinesin, $\theta_{\mathrm{rms}}$ decreases much faster than (b) at high concentration of kinesin.

detachment from the surface. We eliminated this artefact by mixing the same concentration of kinesin (the same used to first introduce kinesins into the device) into the buffer solutions used in the experiment, to maintain a constant surface density of kinesin during the application of shear flow. These free kinesins in the buffer solution prevented the decline of the surface density of kinesin and this method proved to be quite successful. Clearly, in future devices, kinesins should be irreversibly crosslinked to the substrate surface.

Our cantilever beam hypothesis is supported by observations of microtubule tip fluctuations in gliding assays. This is caused by thermal fluctuation (the energy of which is proportional to $k T$, where $k$ is the Boltzmann constant and $T$ is the absolute temperature) that causes random events in individual microtubule translocation and alignment. Similarly, in the presence of shear, a microtubule's trend towards alignment is not always monotonic, as seen in figure 9 . We also note that the late-time $\theta_{\mathrm{rms}}$ appears to asymptotically approach to a non-zero value, and that this asymptotic $\theta_{\mathrm{rms}}$ is larger at higher kinesin concentrations (see figure 10). A similar trend has been also observed in the presence of electric fields [24]

This might seem counterintuitive, but this observation of poorer late-time alignment with higher kinesin density can be explained by scaling arguments. As some length $\Delta L$ of a microtubule tip becomes cantilevered, the deflection of the tip due to Brownian motion is expected to scale as $\left(\Delta L^{3} k T\right)^{1 / 2}$ (see Gittes et al [30]). However, at the same time, the steady deflection due to the viscous drag force scales as $\Delta L^{4}$. Thus, in high-density-kinesin cases, when the typical $\Delta L$ is shorter, the ratio of random deflection to steady deflection is actually larger when $\Delta L$ is shorter, so the range of possible turning angles as the tip moves from kinesin to kinesin is actually larger. This result also implies that microtubule alignment may become extremely slow at very high kinesin concentration. Furthermore, since the microtubules may be detached by extremely high shear stress, one would expect that lower kinesin densities give a wider range of shear stresses available for effective alignment of microtubules.

As for the future of this approach for developing $\mu$ TAS systems powered by biomolecular motors and microtubules, we may need practical, uncomplicated wall shear stress (nanolitre flow rate) control in the microchannel. Since, typically, a head-height difference $(H)$ of $1 \mathrm{~cm}$ between reservoirs can produce a hydrostatic pressure gradient $\left(\Delta P_{\mathrm{ch}}\right)$ of about $100 \mathrm{~Pa}\left(\Delta P_{\mathrm{ch}}=\rho g H, \rho\right.$ is the density of a fluid), from equation (1), the required wall shear stress is determined by the geometry of the microchannel. For instance, the microchannel used in our experiments $(100 \mu \mathrm{m}$ deep, $400 \mu \mathrm{m}$ wide, and $15 \mathrm{~mm}$ long) results in a hydrodynamic resistance of $R_{\mathrm{ch}}=$ $4.94 \times 10^{12} \mathrm{~kg} \mathrm{~m}^{-4} \mathrm{~s}^{-1}$ (see Online Supporting Information available at stacks.iop.org/Nano/18/025101). Therefore, the average wall shear stress $\left(\bar{\tau}_{\mathrm{w}}\right)$ in the microchannel becomes approximately $0.3 \mathrm{~Pa}$. Since the shear stress can be linearly controlled by changing the head-height difference and most $\mu$ TAS systems (including bimolecular motor-driven devices) require loading flows through the microchannel, the shear flowbased microtubule redirection/alignment method will be quite useful for developing biomolecular motor powered, standalone devices.

\section{Conclusion}

Pressure-driven shear flow can be used as an effective method to actively control the direction of microtubules on kinesincoated surfaces. The proposed mechanism appears to be that shear flow imposes viscous drag forces on cantilevered leading ends of the microtubules during translocation. This hypothesis suggests that the kinesin density is a major factor governing both the rate of redirection, and the ultimate statistical degree of alignment-lower kinesin densities lead to both faster and better ultimate alignment of the microtubules. Statistical data on the rate of microtubule alignment, and scaling arguments for the relative importance of random tip fluctuations compared to shear-induced tip deflection, support this hypothesis. As a practical methodology, we discovered that using kinesin-free buffer to flush such systems results in rapid rates of detachment (over a few or a few tens of seconds). We suggest that the desorption rate of kinesin from the surface may be of the order of this timescale, and so kinesin must be added to the flushing solution to prevent this problem. It is hoped these results are useful beyond our interest in microsystem development, e.g. to help understand the effects of shear and flow on microtubule translocation in biological systems. 


\section{Acknowledgment}

This work was funded by NSF Award BES-0428090.

\section{References}

[1] Howard J 2001 Mechanics of Motor Proteins and the Cytoskeleton (Sunderland, MA: Sindauer)

[2] Hunt A J, Gittes F and Howard J 1994 The force exerted by a single kinesin molecule against a viscous load Biophys. $J$. 67 766-81

[3] Kojima H, Muto E, Higuchi H and Yanagida T 1997 Mechanics of single kinesin molecules measured by optical trapping nanometry Biophys. J. 73 2012-22

[4] Meyhöfer E and Howard J 1995 The force generated by a single kinesin molecule against an elastic load Proc. Natl Acad. Sci. 92 574-8

[5] Svoboda K, Schmidt C F, Schnapp B J and Block S M 1993 Direct observation of kinesin stepping by optical trapping interferometry Nature 365 721-7

[6] Bohm K J, Stracke R, Baum M, Zieren M and Unger E 2000 Effect of temperature on kinesin-driven microtubule gliding and kinesin ATPase activity Febs Lett. 466 59-62

[7] Schnitzer M J and Block S M 1997 Kinesin hydrolyses one ATP per 8-nm step Nature $\mathbf{3 8 8} 386-90$

[8] Howard J, Hudspeth A J and Vale R D 1989 Movement of microtubules by single kinesin molecules Nature 342 154-8

[9] Hess H, Bachand G D and Vogel V 2004 Powering nanodevices with biomolecular motors Chem.-Eur. J. $102110-6$

[10] van den Heuvel M G L, De Graaff M P and Dekker C 2006 Molecular sorting by electrical steering of microtubules in kinesin-coated channels Science 312 910-4

[11] Yokokawa R, Takeuchi S, Kon T, Nishiura M, Sutoh K and Fujita H 2004 Unidirectional transport of kinesin-coated beads on microtubules oriented in a microfluidic device Nano Lett. 4 2265-70

[12] Kim T S et al 2003 Biomolecular motors as novel prime movers for microTAS: microfabrication and material issue MicroTAS $133-6$

[13] Gittes F, Meyhöfer E, Baek S and Howard J 1996 Directional loading of the kinesin motor molecule as it buckles a microtubule Biophys. J. 70 418-29

[14] Cheng L J, Kao M T, Meyhöfer E and Guo L J 2005 Highly efficient guiding of microtubule transport with imprinted CYTOP nanotracks Small 1 409-14

[15] Clemmens J, Hess H, Howard J and Vogel V 2003 Analysis of microtubule guidance in open microfabricated channels coated with the motor protein kinesin Langmuir 19 1738-44
[16] Clemmens J, Hess H, Lipscomb R, Hanein Y, Bohringer K F, Matzke C M, Bachand G D, Bunker B C and Vogel V 2003 Mechanisms of microtubule guiding on microfabricated kinesin-coated surfaces: chemical and topographic surface patterns Langmuir 19 10967-74

[17] Dennis J R, Howard J and Vogel V 1999 Molecular shuttles: directed motion of microtubules along nanoscale kinesin tracks Nanotechnology $\mathbf{1 0} 232-6$

[18] Hiratsuka Y, Tada T, Oiwa K, Kanayama T and Uyeda T Q P 2001 Controlling the direction of kinesin-driven microtubule movements along microlithographic tracks Biophys. J. 81 1555-61

[19] Jia L L, Moorjani S G, Jackson T N and Hancock W O 2004 Microscale transport and sorting by kinesin molecular motors Biomed. Microdev. 6 67-74

[20] Lin C T, Kao M T, Kurabayashi K and Meyhöfer E 2006 Efficient designs for powering microscale devices with nanoscale biomolecular motors Small 2 281-7

[21] Bohm K J, Stracke R, Muhlig P and Unger E 2001 Motor protein-driven unidirectional transport of micrometer-sized cargoes across isopolar microtubule arrays Nanotechnology 12 238-44

[22] Prots I, Stracke R, Unger E and Böhm K J 2003 Isopolar microtubule arrays as a tool to determine motor protein directionality Cell Biol. Int. 27 251-3

[23] Stracke R, Bohm K J, Burgold J, Schacht H J and Unger E 2000 Physical and technical parameters determining the functioning of a kinesin-based cell-free motor system Nanotechnology 11 52-6

[24] Kim T S, Kao M-T, Hasselbrink E F and Meyhöfer E 2006 Active alignment of microtubules with electric fields Nano Lett. accepted

[25] Stracke R, Böhm K J, Wollweber L, Tuszynski J A and Unger E 2002 Analysis of the migration behaviour of single microtubules in electric fields Biochem. Biophys. Res. Commun. 293 602-9

[26] Lakämper S, Kallipolitou A, Woehlke G, Schliwa M and Meyhöfer E 2003 Single fungal kinesin motor molecules move processively along Microtubules Biophys. $J$. 84 1833-43

[27] Lakämper S and Meyhöfer E 2005 The E-hook of tubulin interacts with kinesin's head to increase processivity and speed Biophys. J. 89 3223-34

[28] Jo B H, Van Lerberghe L M, Motsegood K M and Beebe D J 2000 Three-dimensional micro-channel fabrication in polydimethylsiloxane (PDMS) elastomer $J$. Microelectromech. Syst. $976-81$

[29] White F M 2001 Viscous Fluid Flow 3rd edn (New York: McGraw-Hill) pp 110-8

[30] Gittes F, Mickey B, Nettleton J and Howard J 1993 Flexural rigidity of microtubules and actin-filaments measured from thermal fluctuations in shape J. Cell Biol. 120 923-34 